\title{
SOFTWARE SOLUTION INTEGRATED IN THREE- DIMENSIONAL TECHNOLOGIES FOR PREOPERATIVE PLANNING OF TIBIAL PLATEAU FRACTURES
}

\author{
Flaviu Moldovan ${ }^{1}$, Adrian Gligor ${ }^{2}$ and Tiberiu Bataga ${ }^{3}$ \\ ${ }^{\prime}$ IOSUD Doctoral School, "George Emil Palade" University of Medicine, Pharmacy, Science, and Technology \\ of Targu Mures \\ 38, Gh. Marinescu street, 540139 Targu Mures, Romania \\ ${ }^{1}$ moldovan.flaviu95@yahoo.com \\ ${ }^{2}$ Biomedical Research Center, "George Emil Palade" University of Medicine, Pharmacy, Science, and \\ Technology of Targu Mures, \\ 2adrian.gligor@umfst.ro \\ 38, Gh. Marinescu street, 540139 Targu Mures, Romania \\ ${ }^{3}$ Department of Orthopedics-Traumatology, "George Emil Palade" University of Medicine, \\ Pharmacy, Science, and Technology of Targu Mures, \\ 38, Gh. Marinescu street, 540139 Targu Mures, Romania \\ 3rtbataga@gmail.com
}

\begin{abstract}
Background: Planning in orthopedic surgery can be supported by various virtual reconstruction and tri-dimensional (3D) segmentation programs, but this topic requires further study to identify software solutions that make the process duration more efficient. Objective: To validate $3 D$ software solutions that integrates $3 D$ technologies for patientspecific applications in orthopedics in order to minimize the extent of surgery. Method: We have used the Democratiz3D software solution for patient-specific modeling and surgical planning. Results: Validation of the proposed methodology was performed for the preoperative planning of a 28-year-old male patient who had a Schatzker type II tibial plateau fracture. Conclusion: The 3D planning capabilities of the software solution are a valuable tool for surgeons in exploring the nature of fractures and formulating an appropriate surgical plan which creates perspectives for personalized surgery.
\end{abstract}

Key words: orthopedic surgery, tibial fracture, three-dimensional technologies, image acquisition

\section{Introduction}

Optimized radiography and computed tomography (CT) imaging are essential for individualized therapy planning [1]. It demands excellent resolution for the delineation of complex fractures and joint alignment after trauma [2], which intra-operative can be achieved with mobile special C-arms [3].
Reconstruction of tibial plateau fractures can be supported by $3 \mathrm{D}$ technologies [4], in order to minimize surgery [5], as both visual and printed models provide tactile feedback and in-depth information about anatomical and pathological conditions [6,7]. Threedimensional (3D) technologies allow for optimal results in reducing fractures [8], identifying the correct alignment [9], fixing with screws [10,11], reducing the

(C) 2021 Published by University Press. This is an open access article under the CC BY-NC-ND license (http://creativecommons.org/licenses/CC-BY-4.0/) 
duration of surgery, blood loss and radiation exposure [12], as well as and improving mechanical alignment in total knee replacement $[13,14,15]$, which is facilitated by patient-specific implants that fit perfectly and provide unlimited geometric mobility [16]. Post mortem computed tomography can also be used to assess complex trauma to victims [17].

Planning in orthopedic surgery can be supported by various virtual reconstruction and $3 \mathrm{D}$ segmentation programs, such as $\operatorname{VoXim}^{\circledR}$ [18], or can be implemented as an OsiriX plugin [19]. But this topic requires further study to identify software solutions that make the process more efficient. Optimization of post-processing algorithms, rather than modifications of image acquisition, may increase the image quality [20].

The hypothesis of the study is to show that the use of three-dimensional technologies is effective in surgical planning for the reconstruction of tibial plateau fractures.

The aim of this study is to validate 3D software solutions that integrate 3D technologies for patientspecific applications in orthopedics that are used in preoperative planning of tibial plateau fractures in order to minimize the extent of surgery.

\section{Materials and Methods}

The use of three-dimensional (3D) technologies in orthopedic surgery is of growing scientific interest, especially in the last decade, and digitization offers a number of tools to increase the volume, quality and accuracy of orthopedic research results. The data from the medical images of the patients are processed with the support of specialized software products, which allow the identification of the analyzed tissues and the extraction of specific information.

The acquisition of 3D images involves the use of various available techniques, computed tomography and magnetic resonance imaging, which can provide images that constitute raw data for later use in $3 \mathrm{D}$ processing.

Each image is made up of a multitude of shades of gray, which consist of collections of pixels or voxels at a certain resolution. For example, in computed tomography, the gray scale is expressed in Hounsfield units, in which metallic materials have a value of 3072 Hounsfield units, water has a value of 0 Hounsfield units, while air has a negative value of -1024 Hounsfield units. Therefore, high-density structures appear dark ("black") and low-density structures appear light ("white"). By selecting a density threshold of a certain value, the desired structures can be highlighted, which allows the differentiation of the tissues that make up the tomographic image. This process of identifying and classifying tissues is called segmentation, which consists of isolating all the pixels and / or voxels of the analyzed component. It is known that scanning acquisition settings affect Hounsfield units (HU) of the CT voxels [21].
In order to acquire images and achieve threedimensional design, we have identified computer programs that can process $3 \mathrm{D}$ images for orthopedic modeling, respectively tissue segmentation and reconstruction.

The software solution we used is Democratiz3D, from the Embodi3d platform [22], which allows patient-specific modeling and subsequently, surgical planning. Because the $3 \mathrm{D}$ reconstruction process requires significant memory resources and computing power in most situations, we used a Supermicro AS1014S-WTRT computing system equipped with an AMD EPYC 7702P 64-Core processor and 512 GB RAM, as well as tools and services in cloud computing.

Democratiz3D is an intuitive, easy-to-use solution that provides good results for $3 \mathrm{D}$ reconstruction problems, and especially for obtaining 3D models that are to be printed by rapid prototyping.

In order to perform the $3 \mathrm{D}$ reconstruction, it is necessary to go through three steps: (1) pre-format the data; (2) 3D primary reconstruction; (3) postprocessing of the $3 \mathrm{D}$ reconstruction model.

\section{(1) Data pre-formatting}

At this stage, the data is converted to the format accepted by the Democratiz3D cloud application, namely Nearly Raw Raster Data (NRRD), which has the advantage of compactly organizing the imaging data, allowing their anonymization and sharing the results.

The easiest way to convert from Digital Imaging and Communications in Medicine (DICOM) format to NRRD can be done with the Slicer application, in two steps, respectively the import of DICOM format in the workspace and then their export in the desired format (fig. 1).

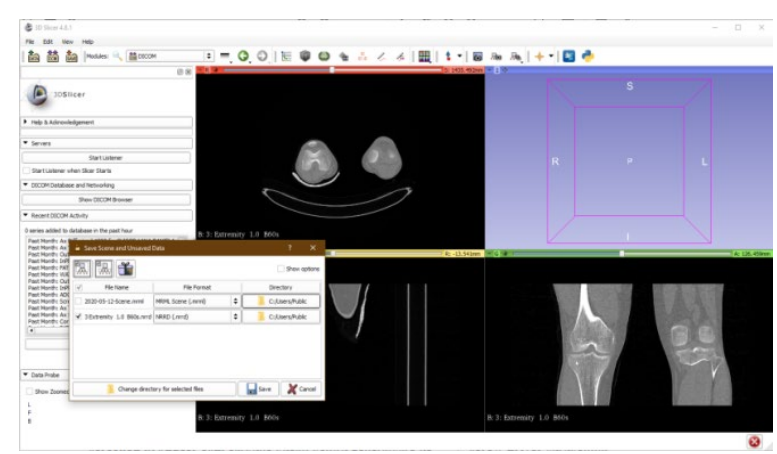

Fig. 1: Conversion of data from DICOM format to NRRD.

In the $3 \mathrm{D}$ reconstruction phase, NRRD data was loaded into the preset category selected for reconstruction. To improve the quality of the surfaces generated by including the imaging information in the $3 \mathrm{D}$ reconstruction model, the threshold level was parameterized. It was necessary to post-process the 3D reconstruction model in order to correct the surface and remove unnecessary components. The processing was done with Meshmixer, which is a Computer Aided 
Design (CAD) application. At the end, the data were prepared in $\mathrm{CAD}$ format.

(2) $3 \mathrm{D}$ primary reconstruction

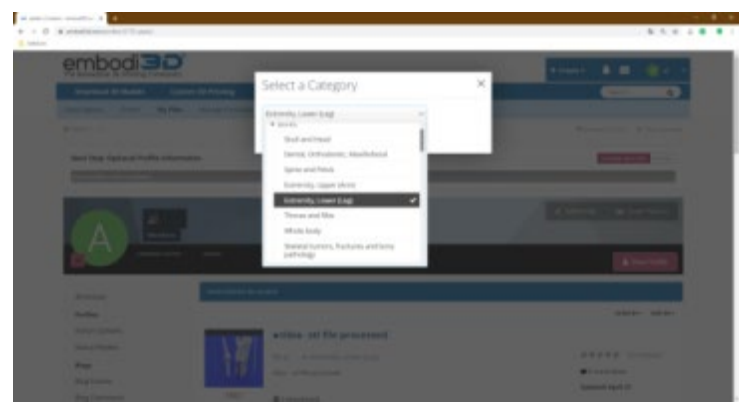

Fig. 2: Reconstruction category selection in Democratiz3D platform.

At this stage the Democratiz3D service is used by choosing the appropriate category (fig. 2) and uploading the data in NRRG format, in the category selected for reconstruction (fig. 3).

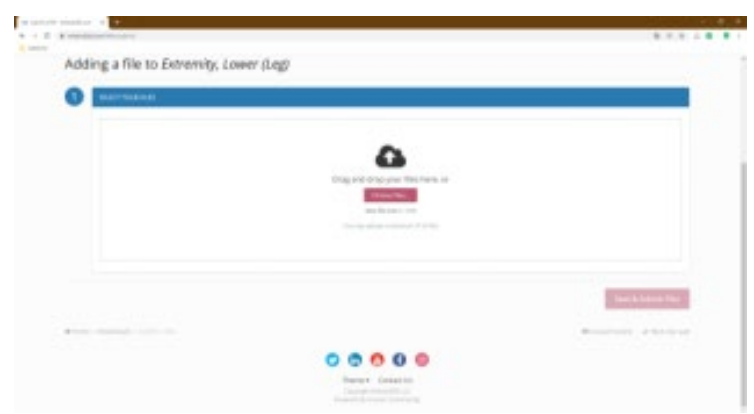

Fig. 3: Data upload for reconstruction in Democratiz3D platform.

The quality of the generated surfaces is parameterized according to the requirements, through the threshold level that allows the inclusion of imaging information in the 3D reconstruction model (fig. 4).

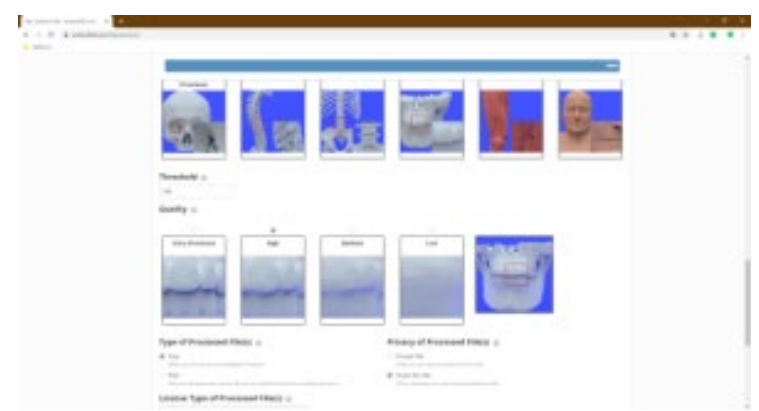

Fig. 4. Advanced parameterization options in 3D primary reconstruction with Democratiz3D.

After processing, the final result in CAD format is downloaded from the web interface of the application (fig. 5).

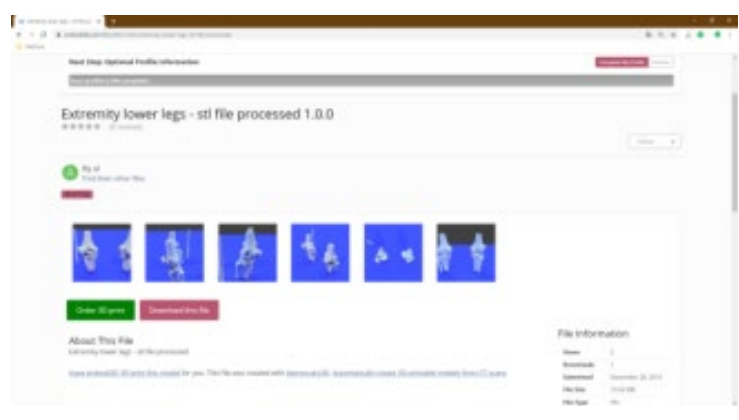

Fig. 5 . The result of the 3D primary reconstruction with Democratiz3D.

(3) Post-processing of the $3 \mathrm{D}$ reconstruction model

The result of the reconstruction with Democratiz3D requires further processing consisting of surface corrections or the removal of unnecessary components. These can be achieved by processing in CAD applications such as Mesodesmixer from Autodesk (fig. 6) or FreeCAD (fig. 7).

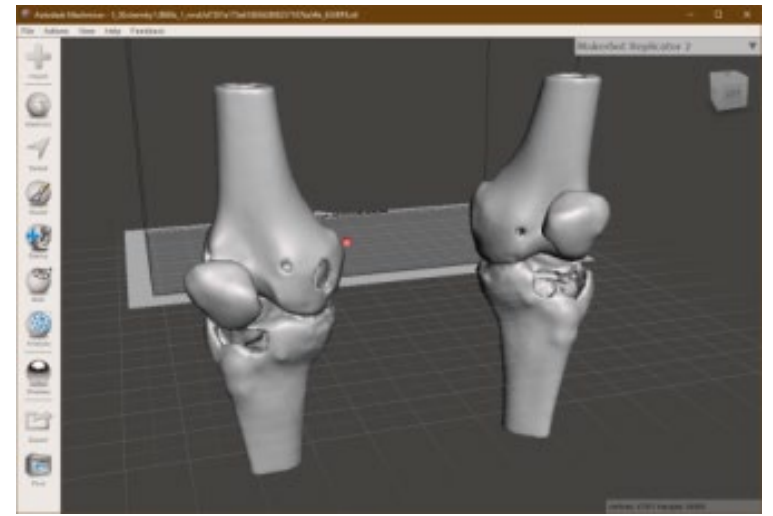

Fig. 6. Model obtained in Democratiz3D after processing with Meshmixer.

The use of the tools mentioned above allows obtaining models that can be converted to "solid" type representations. In the case of complex surfaces, processing can be difficult and time consuming.

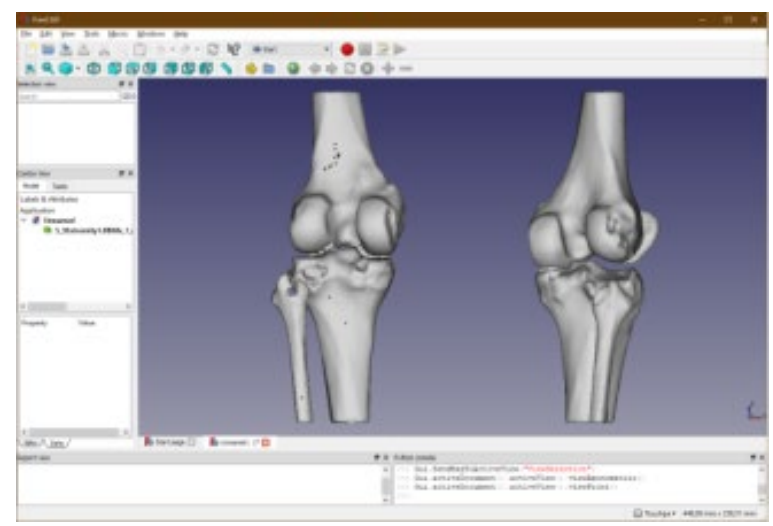

Fig. 7. Model obtained in Democratiz3D after processing with FreeCAD.

In this way, preoperative planning can be customized and the surgical treatment of tibial plateau 
fractures can be customized.

\section{Results}

This paragraph presents the results of the $3 \mathrm{D}$ reconstruction of the tibial plateau, using 3D technologies in the visualization and surgical planning stages.

This allows a better understanding of the reduction, validated in practice for the case of a tibial plateau fracture that we collected from the Emergency Hospital U.P.U.-S.M.U.R.D. Târgu Mureș.

The 28-year-old male patient was sent to the orthopedics department, based on the initial clinical examination, diagnosis and imaging examination performed in the emergency department. Tibia scans were performed with a CT OPTIMA $580 \mathrm{~W}$. The fracture was classified according to the Schatzker classification as type II. In fig. 8 is presented the DICOM file and in fig. 9 is presented the analysis of the DICOM file with the RadiAnt DICOM Viewer software of the patient's fracture included in study.

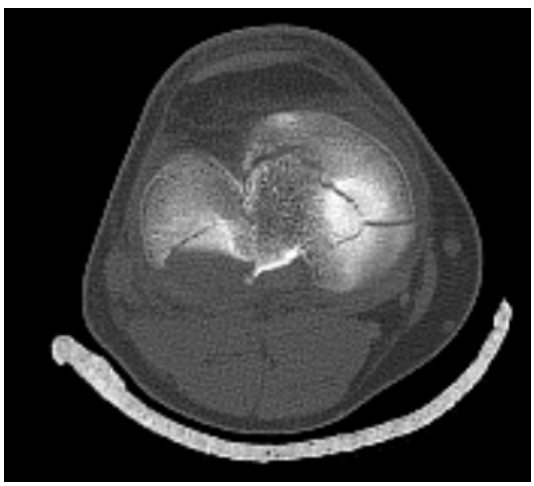

Fig. 8. DICOM image of the Schatzker type II fracture in the right leg of the patient included in the study.

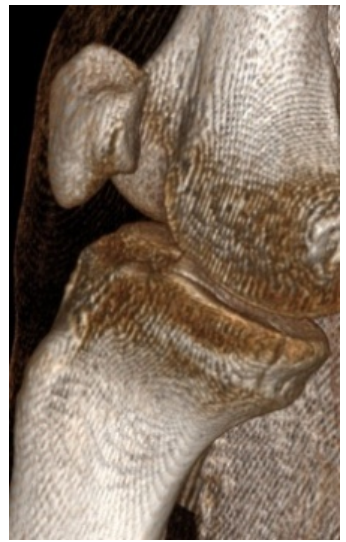

Fig. 9. RadiAnt image of the Schatzker type II fracture in the right leg of the patient included in the study.

Figures 10 and 11 show the reconstruction results with 18 points of $3 \mathrm{D}$ connectivity, from whose analysis it can be concluded that the choice of reconstruction parameters is important, because it can significantly influence the details and quality of the generated surfaces.

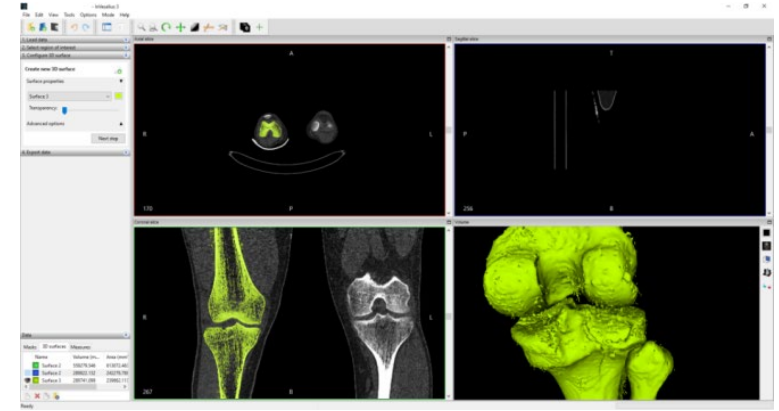

Fig. 10. Profile detail reconstruction view with 18 3D connectivity points showing high quality surface.

With the support of the tools presented in this study, successful 3D segmentation and reconstruction was obtained in the case of the patient with tibial plateau fracture. The time elapsed from the beginning to the end of the case analysis was recorded, and the time required for type II fracture planning was 103 $\min$.

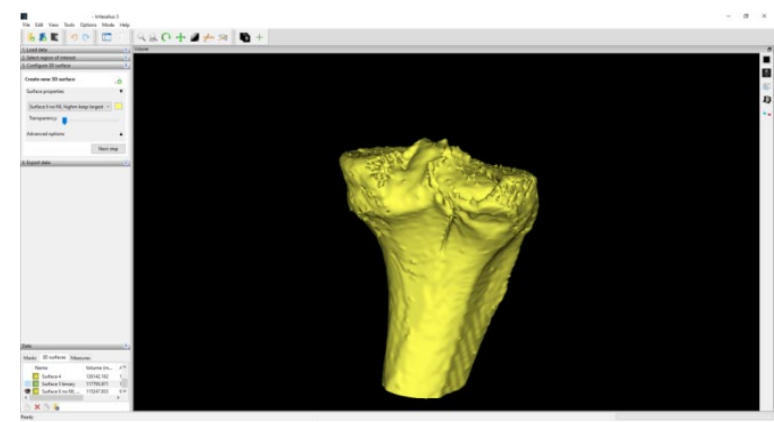

Fig. 11. Profile detail reconstruction view with 18 points of high quality surface 3D connectivity - Schatzker Fracture type II.

\section{Discussion}

In this study, a 3D reconstruction method based on Democratiz3D software was presented. If we analyze it from the point of view of the processing flow, it is characterized by the following important properties:

- Allows easy use and a learning curve without difficulty for the preprocessing part in Democatiz3D;

- It employs cloud processing resources, which facilitates preprocessing;

- Modification is intuitive for reconstruction parameters;

- Access is free for standard models;

- Requires a large number of applications to obtain a final 3D reconstruction model and consequently requires knowledge of how to operate several IT tools;

- Democratiz3D is designed to make printable 3D models, generates monobloc models, and in the case of the coexistence of multiple morphological components, they are difficult or impossible to separate in real forms of representation by using post-processing tools;

- Providing the result of processing with Democratiz3D is platform dependent, which 
makes the generation of 3D models more time consuming;

- Testing the comparative reconstruction with several parameters is difficult, because it requires the resumption of the whole process from the raw data loading stage;

- The method requires laborious work to correct and complete the surfaces of the reconstructed model.

The implications of the study in the field consist in:

- Identification of computer programs available free of charge on Internet at present and presentation of the sequence of their use in order to process $3 \mathrm{D}$ images for orthopedic modeling in personalized surgical treatment;

- The 3D capabilities of the program used allow the user to manipulate and see the fracture site from any plane, without subjecting the patient to awkward manipulation while taking radiographic images. This information can help the surgeon to elaborate the surgical plan, as well as to compare it with the postoperative results;

- The use of 3D images in tibial plateau fractures helps the surgeon in planning the surgical approach: a) they allow the surgeon to better understand the nature of the fracture and to identify the difficult reductions of the fragment; b) allow a better identification of the independent fracture fragments; c) careful reconstruction of the articular line; d) reducing the risk of post-traumatic osteoarthritis.

The use of this method based on 3D software has some disadvantages. The time required for $3 \mathrm{D}$ segmentation reconstruction and analysis may be a discouraging factor for its current use in the clinical setting, and the amount of new information obtained in less complicated fractures may not be worth the time invested in planning preoperative $3 \mathrm{D}$ reconstruction.

Running the software used in the current study required relatively long timeframes, and a suggestion for the future is to identify newer versions of this software that should reduce the time required for planning, while increasing segmentation and cleaning facilities of images. Also, following randomized studies, it should be determined whether the clinical outcome is worth the time.

The investigated case of tibial plateau fracture allowed the validation of the software solution for segmentation and $3 \mathrm{D}$ reconstruction of the tibial plateau, confirming the research hypothesis on improving the quality of the tibial plateau reconstruction process by customizing surgical treatment with the support of 3D technologies. There are some reservations about the length of the trial, and following randomized studies, it should be determined whether the clinical outcome is worth the time.

The results are consistent with other studies in the literature reported by Suero et al [18], which use the VoXim1 planning software and indicate average times for $3 \mathrm{D}$ virtual planning of tibial plateau fractures of
$174.8 \mathrm{~min}$, with an average time required for virtual 3D planning of type B fractures of $96.5 \mathrm{~min}$ and an average time required for planning type $\mathrm{C}$ fractures of $227 \mathrm{~min}$.

\section{Conclusion}

The 3D planning capabilities of the software solution developed and used in this study are a valuable tool for surgeons in exploring the nature of tibial plateau fractures and formulating an appropriate surgical plan that opens up perspectives on:

- Surface alignment - the coordinates of the points on the fracture surfaces are described mathematically and alignment methods can be used, which allow preoperative simulation of the desired alignment;

- Design and 3D printing of screw fixation guides for tibial fracture fragments;

- Establishing by preoperative simulation the optimal direction of the percussive screws for fixing the bone fragments;

- Strength calculations of bone fragments fixed by screws.

It can be concluded that virtual modeling with the help of computer and the physical production of anatomical replicas and guides can be combined as needed, offering many advantages for the realization of personalized therapy.

The creation of 3D anatomy visualizations or 3D anatomical replicas identical to the actual organs assists surgeons and is revolutionizing preoperative planning for many orthopedic procedures from various cases of complex trauma to elective orthopedic surgery, which confirms the hypothesis formulated in this study on the efficiency of using three-dimensional technologies in surgical planning for the reconstruction of tibial plateau fractures.

Institutional Review Board Statement: The study was conducted according to the guidelines of the Declaration of Helsinki, and approved by the Ethics Committee of Emergency County Hospital Târgu Mureș (protocol code 2Ad.6108/02.03.2021).

Informed Consent Statement: Informed consent was obtained from the patient involved in the study.

Conflicts of Interest: The authors declare no conflict of interest.

\section{References}

[1] Grunz, J.P., Gietzen, C.H., Schmitt, R. and Prommersberger, K.J. (2018), Distale Radiusfrakturen: Update zur Bildgebung [Distal radius fractures: Update on imaging]. Radiologe, vol. 58(2), pp. 159-174.

[2] Johnson, P.T., Fayad, L.M. and Fishman. E.K. (2006), Sixteen-slice CT with volumetric analysis of foot fractures. Emerg Radiol., vol. 12(4), pp. 171-6.

[3] Keil, H., Beisemann, N., Swartman, B., Vetter, S.Y., Grützner, P.A. and Franke, J. (2018), Intra- 
operative imaging in trauma surgery. EFORT Open Rev., vol. 3(10), pp. 541-549.

[4] Moldovan, F., Gligor, A. and Bataga T. (2020), Integration of Three-dimensional Technologies in Orthopedics: A Tool for Preoperative Planning of Tibial Plateau Fractures. Acta Inform Med., vol. 28(4), pp. 278-282.

[5] Weimann, A., Heinkele, T., Herbort, M., Schliemann, B., Tetersen, W. and Raschke, M.J. (2013), Minimally invasive reconstruction of lateral tibial plateau fractures using the jail technique: A biomechanical study. $B M C$ Musculoskeletal Disorders, vol. 14: 120.

[6] Mitsouras, D., Liacouras, P., Imanzadeh, A., Giannopoulos, A.A., Cai, T., Kumamaru, K.K, et al. (2015), Medical 3D printing for the radiologist. Radiographics, vol. 35(7), pp. 19651988.

[7] Bizzotto, N., Tami, I., Santucci, A., Adani, R., Poggi, P., Romani, D., et al. (2016), 3D Printed replica of articular fractures for surgical planning and patient consent: A two years multi-centric experience. 3D Printing in Medicine, vol. 2(2), pp. 1-6.

[8] Moldovan, F., Gligor, A. and Bataga, T. (2021), Structured Integration and Alignment Algorithm: A Tool for Personalized Surgical Treatment of Tibial Plateau Fractures. J. Pers. Med., vol. 11: 190.

[9] Vlachopoulos, L., Székely, G., Gerber, C. and Fürnstahl, P. (2018), A scale-space curvature matching algorithm for the reconstruction of complex proximal humeral fractures. Med Image Anal., vol. 43, pp. 142-156.

[10] Huang, H., Hsieh, M.F., Zhang, G., Ouyang, H., Zeng, C., Yan, B., et al. (2015), Improved accuracy of 3D-printed navigational template during complicated tibial plateau fracture surgery. Australas Phys Eng Sci Med., vol. 38(1), pp. 109-117.

[11] Huang, H., Zhang, G., Ouyang, H., Yang, Y., Wu, $\mathrm{Z}$., $\mathrm{Xu}, \mathrm{J}$., et al. (2015), Internal fixation surgery planning for complex tibial plateau fracture based on digital design and 3D printing. J South Med Univ., vol. 35(2), pp. 218-222.

[12] Giannetti, S., Bizzotto, N., Stancati, A. and Santuci, A. (2017), Minimally invasive fixation in tibial plateau fractures using an pre-operative and intra-operative real size $3 \mathrm{D}$ printing. Injury, vol. 48(3), pp. 784-788.

[13] Gemalmaz, H.C., Sariyılmaz, K., Ozkunt, O., Sungur, M., Kaya, I. and Dikici, F. (2019), Postoperative mechanical alignment analysis of total knee replacement patients operated with 3D printed patient-specific instruments: a prospective cohort study. Acta Orthop Traumatol Turc., vol. 53(5), pp. 323-328.

[14] Cucchi, D., Menon, A., Aliprandi, A., Soncini, G., Zanini, B., Ragone, V., et al. (2019), Patientspecific instrumentation affects rotational alignment of the femoral component in total knee arthroplasty: A prospective randomized controlled trial. J Orthop Surg., vol. 11(1), pp. 75-81.

[15] Vaishya, R., Vijay, V., Birla, V.P. and Agarwal AK. (2016), Computerized tomography based "patient-specific blocks" improve postoperative mechanical alignment in primary total knee arthroplasty. World Journal of Orthopedics, vol. 7(7), pp. 426-433.

[16] Javaid, M. and Haleem, A. (2018), Additive manufacturing applications in orthopedics: a review. J Clin Orthop Trauma, vol. 9(3), pp. 202-206.

[17] Di Paolo, M., Maiese, A., dell'Aquila, M., Filomena, C., Turco, S., Giaconi, C. and Turillazzi, E. (2020), Role of post mortem CT (PMCT) in high energy traumatic deaths. Clin Ter., vol. 171(6):e490-e500.

[18] Suero, E.M., Hufner, T., Stubig, T., Krettek, C. and Citak, M. (2010), Use of a virtual 3D software for planning of tibial plateau fracture reconstruction. Injury, vol. 41(6), pp. 589-591.

[19] Ribeiro, J., Alves, V., Silva, S. and Campos, J. (2015), A 3D computed tomography based tool for orthopedic surgery planning. Published in: Developments in Medical Image Processing and Computational Vision. Springer International Publishing. Editors: João Manuel R. S. Tavares, Renato Natal Jorge, pp.121-138.

[20] Graul, I., Marintschev, I., Rausch, S., Eckart, N., Hofmann, G.O. and Gras, F. (2019), Effect of different multiplanar reformation algorithms on image quality of intraoperative threedimensional fluoroscopy. J Hand Surg Eur., vol. 44(7), pp. 738-744.

[21] Giambini, H., Dragomir-Daescu, D., Nassr, A., Yaszemski, M.J. and Zhao, C. (2016), Quantitative Computed Tomography Protocols Affect Material Mapping and Quantitative Computed Tomography-Based Finite-Element Analysis Predicted Stiffness. J Biomech Eng., vol. 138(9):0910031-7.

[22] ***https://www.embodi3d.com/ democratiz3D/. 